\title{
Relación entre consumo de sustancias y rasgos esquizotípicos en adolescentes escolarizados
}

\section{Relationship between substance use and schizotypal traits in school-aged adolescents}

\author{
Ma de los Ángeles Sánchez-García*, Javier Ortuño-Sierra*, Mercedes Paino**, \\ Eduardo Fonseca-PEdrero***** \\ * Departamento de Ciencias de la Educación. Universidad de La Rioja, Logroño. España. \\ ** Departamento de Psicología. Universidad de Oviedo, Oviedo. España. \\ *** Centro de Investigación Biomédica en Red de Salud Mental (CIBERSAM), Oviedo. España.
}

\section{Resumen}

Objetivo: analizar la posible relación entre el consumo de sustancias psicoactivas (en concreto, tabaco, alcohol y cannabis) y los rasgos esquizotípicos en una muestra representativa de adolescentes escolarizados de la población general. Método: diseño transversal descriptivo tipo encuesta. La muestra se compuso por 1.588 estudiantes $(M=16,13$ años; $D T=1,36 ; 739$ varones, $46,5 \%)$, seleccionados mediante muestreo aleatorio estratificado por conglomerados. Los instrumentos administrados fueron el Cuestionario Oviedo para la Evaluación de la Esquizotipia, el Cuestionario de Consumo de Sustancias Modificado, el Cuestionario de Capacidades y Dificultades, el Penn Matrix Reasoning Test, la Family Affluence Scale-II y la Escala Oviedo de Infrecuencia de Respuesta. Resultados: una vez controlado el efecto de múltiples covariables (género, edad, cociente intelectual, nivel socio-económico, psicopatología previa y consumo de sustancias), los consumidores de alcohol comparados con los no consumidores, informaron de mayores puntuaciones medias en la dimensión Desorganización Social. Los consumidores de tabaco comparados con los no consumidores, informaron de mayores puntuaciones medias en las dimensiones de esquizotipia de Anhedonia y Desorganización Social. Con respecto al consumo de cannabis, no se hallaron diferencias estadísticamente significativas entre consumidores y no consumidores en las dimensiones esquizotípicas. Conclusiones: controlando el efecto de múltiples variables de confundido, los adolescentes consumidores de tabaco y alcohol -según su frecuencia- informaron de mayores puntuaciones en rasgos esquizotípicos. Futuros estudios podrían continuar analizando el papel del consumo de sustancias en la aparición de trastornos mentales, como la psicosis, mediante nuevas metodologías como la evaluación ambulatoria.

Palabras clave: Adolescencia; Consumo sustancias; Drogas; Esquizotipia; Rasgos esquizotípicos.

\begin{abstract}
Goal: The main goal of the present study was to analyze the relationship between substance use (tobacco, alcohol, cannabis) and schizotypal traits in a representative sample of school-aged adolescents from the general population. Method: cross-sectional, descriptive survey 1,588 students $(M=16.13$ years, $S D=1.36), 739$ male $(46.5 \%)$, selected by random stratified cluster sampling, participated in the study. The instruments administered were the Oviedo Questionnaire for Schizotypy Assessment, the Modified Substance Use Questionnaire, the Strengths and Difficulties Questionnaire, the Penn Matrix Reasoning Test, the Family Affluence Scale-II, and the Oviedo Infrequency Scale. Results: Controlling for the effects of multiple covariates (gender, age, IQ, socio-economic level, psychopathology and consumption), the results showed that compared with non-users, alcohol drinkers reported higher average scores in the Social Disorganization dimension. Cigarette smokers, differentiated by frequency, reported higher average scores in the Anhedonia and Social Disorganization dimensions than non-smokers. No statistically significant differences between users and non-users of cannabis were found in terms of schizotypal traits. Conclusion: controlling for the effects of multiple covariates, adolescents who use tobacco and alcohol reported higher scores - depending on frequency of use - in schizotypal traits. Future studies should continue to analyze the role of substance use in individuals at risk of psychosis and determine its role in the transition to serious mental disorders using new methodologies such as ambulatory assessment.
\end{abstract}

Keywords: Adolescence; Substance consumption; Drugs; Schizotypy; Schizotypal traits.

Recibido: Julio 2018; Aceptado: Julio 2019.

Enviar correspondencia a: Eduardo Fonseca-Pedrero.

Universidad de La Rioja. Dpto. Ciencias de la Educación. C/ Luis de Ulloa, 2. 26004 Logroño-España. Tlf: +34 941299236. Fax +34 941 299 333. E-mail: eduardo.fonseca@unirioja.es. 


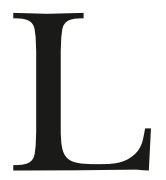

a esquizotipia se define como la predisposición latente a los trastornos del espectro esquizofrénico (Meehl, 1962). Los modelos etiológicos más actuales consideran que dicha vulnerabilidad a la psicosis se expresa a través de un continuo de gravedad, que oscila desde la expresión no clínica (rasgos esquizotípicos, experiencias psicóticas atenuadas), pasando por el nivel subumbral (síntomas psicóticos atenuados) hasta llegar al nivel clínico de psicosis y necesidad de tratamiento (Barrantes-Vidal, Grant y Kwapil, 2015; Fonseca-Pedrero, 2018; Fonseca-Pedrero y Debbané, 2017; Fumero, Marrero y Fonseca-Pedrero, 2018). Las experiencias psicóticas y los rasgos esquizotípicos se encuentran distribuidos normalmente en la población general, sin estar necesariamente asociados a malestar, tratamiento y/o discapacidad (Linscott y Van Os, 2013; Van Os, Linscott, Myin-Germeys, Delespaul y Krabbendam, 2009). Por ejemplo, las tasas de prevalencia de experiencias psicóticas atenuadas es del $17 \%$ en niños de 9 a 12 años, y del 7,5\% entre adolescentes de 13 a 18 años (Kelleher, Connor y Clarke, 2012). Además, tanto las experiencias psicotiformes como los rasgos esquizotípicos son considerados como marcadores de vulnerabilidad para los trastornos del espectro psicótico en general, y para la esquizofrenia en particular (Debbané et al., 2015). Por lo tanto, su análisis puede permitir comprender los posibles mecanismos etiológicos involucrados en la transición a un cuadro psicótico, de cara al establecimiento de tratamientos preventivos (Fonseca-Pedrero e Inchausti, 2018).

Desde el modelo de propensión-persistencia-discapacidad de la psicosis (Van Os et al., 2009) los rasgos esquizotípicos o las experiencias psicotiformes pueden reflejar una vulnerabilidad conductual subyacente que aumenta el riesgo de trastorno psicótico (Ericson, Tuvblad, Raine, Young-Wolff y Baker, 2011; Morton et al., 2017; Shakoor et al., 2016) y que puede estar influenciada por factores como, por ejemplo, el trauma infantil (Abajobir et al., 2017; Arseneault et al., 2011), las autolesiones y comportamientos suicidas, estados afectivos negativos (Fonseca-Pedrero y Debbané, 2017; Najolia, Buckner y Cohen, 2012) y/o por el consumo de sustancias (Hides et al., 2009; Malone, Hill y Rubino, 2010; Saha et al., 2011; Schubart et al., 2011). En este sentido, los factores genéticos podrían determinar la sensibilidad específica de cada individuo a diferentes factores de riesgo ambiental (p. ej., complicaciones obstétricas, consumo de cannabis, experiencias de trauma) y/o psicológico (p. ej., esquemas cognitivos disfuncionales, hiper-reflexibilidad), de manera que los individuos más vulnerables tendrían más riesgo de desarrollar psicosis o algunos de sus síntomas cuando se exponen a uno o varios factores de riesgo. Del mismo modo, múltiples impactos ambientales en diferentes momentos temporales podrían conducir a que las experiencias psicóticas atenuadas y los rasgos esquizotípicos en personas vulnerables se vuelvan persistentes, sobrepasando el umbral clínico y generando una mayor probabilidad de discapacidad y necesidad de tratamiento (Fonseca-Pedrero, 2018; Linscott y Van Os, 2013).

En las últimas décadas se han incrementado considerablemente las investigaciones que examinan la relación entre el consumo de drogas y problemas de salud mental (Casajuana Kögel, López-Pelayo, Balcells-Olivero, Colom y Gual, 2018; Cohn, Johnson, Ehlke y Villanti, 2016; Gonzalvo, Barral y Roncero, 2011; Rial et al., 2018). Uno de los grupos de trastornos mentales que mayor atención ha concitado ha sido el del espectro de psicosis. En este sentido, diferentes estudios de meta-análisis o de revisión constatan la relación entre el consumo de cannabis y el fenotipo psicótico clínico y subclínico (Fonseca-Pedrero, Lucas-Molina, Pérez-Albéniz, Inchausti y Ortuño-Sierra, 2020; Kraan et al., 2016; Large, Sharma, Compton, Slade y Nielssen, 2011; Marconi, Di Forti, Lewis Murray y Vassos, 2016; Moore et al., 2007; Szoke et al., 2014). Por ejemplo, Marconi et al. (2016), encontraron que los altos niveles de consumo de cannabis aumentan el riesgo de psicosis, confirmando una relación dosis-respuesta entre el nivel de uso y el riesgo de psicosis. En otro estudio, Saha et al. (2011) encontraron que los consumidores de cannabis con una edad temprana (16 años o menos) tenían aproximadamente diez veces más posibilidades de mostrar experiencias delirantes que los no consumidores.

Trabajos previos han analizado la relación entre el consumo de cannabis y los rasgos esquizotípicos, sugiriendo que un mayor consumo de cannabis se asocia con puntuaciones mayores en la dimensión Positiva (Distorsión de la Realidad) (Cohen, Buckner, Najolia y Stewart, 2011; Hides et al., 2009; Schubart et al., 2011), si bien es cierto que hay estudios que no han encontrado una asociación estadísticamente significativa (Barkus, 2008; Van Gastel et al., 2012). Además, para la dimensión Negativa, los resultados no son convincentes: algunos estudios encuentran una asociación entre mayor consumo con puntuaciones más altas en anhedonia (Cohen et al., 2011; Schubart et al., 2011; Verdoux y Van Os, 2002), mientras que otros estudios no reflejan tal asociación (Hides et al., 2009; Najolia et al., 2012; Schubart et al., 2011). Por ejemplo, Najolia et al. (2012), en su estudio con adultos jóvenes no clínicos, desvelaron que el consumo de cannabis estaba asociado con tasas más altas de rasgos esquizotípicos positivos y desorganizados, pero no con los rasgos esquizotípicos negativos.

Por otra parte, el policonsumo es lo más habitual entre los consumidores de drogas, con unos efectos adversos que repercuten en factores cognitivos, afectivos y comportamentales de manera que aumenta el riesgo de problemas de salud mental (Moreno, 2018). El alcohol y el tabaco se consideran la "puerta de entrada" a otras drogas ilegales (Vázquez y Becoña, 2000). La literatura también señala que los jóvenes que realizan un consumo intensivo de alcohol tienen mayor probabilidad de verse implicados en con- 
ductas de riesgo tales como violencia, actividad sexual no planificada, absentismo escolar, conducción imprudente (Maturana, 2011); de suicidio (Pérez Gálvez, 2015); y binge drinking (Sendino et al., 2016). Así, diversos estudios encontraron que los adolescentes que eran más vulnerables al alcohol, nicotina y cannabis podían tener mayor riesgo clínico (Auther et al., 2012; Buchy, Perkins, Woods, Liu y Addington, 2014; Fonseca-Pedrero, Ortuño-Sierra, Paino y Muñiz, 2016; Fumero, Santamaría y Navarrete, 2009). Por ejemplo, en una muestra de estudiantes se constató que las puntuaciones de esquizotipia correlacionaban positivamente con el consumo de alcohol y cannabis (García Montes, Zaldívar Basurto, Moreno Montoya y Flores Cubos, 2013). Analizando los rasgos esquizotípicos y el tabaquismo, se encontró que individuos con esquizotipia tenían el doble de probabilidades de fumar y los fumadores reportaron síntomas más graves de desorganización y síntomas esquizotípicos negativos menos graves (Stewart, Cohen y Copeland, 2010).

Además, como demuestra la Encuesta sobre Uso de Drogas en Enseñanzas Secundarias en España (ESTUDES 2016-17) en el año anterior, el 75,6\% de estos escolares había consumido alcohol, un 34,7\% había fumado tabaco y el 26,3 \% había consumido cannabis. En dicha encuesta, un dato significativo fue que la edad media de inicio de consumo de estas sustancias se retrasa a los 14 años. Datos recientes indican que el porcentaje de consumidores problemáticos de cannabis es del 13,3\% (Moreno, 2018). De por sí, la prevalencia del consumo de tabaco, alcohol y cannabis entre los jóvenes es elevada y se asocia con morbi-mortalidad y múltiples costes personales y socio-sanitarios.

El consumo de sustancias es un factor de riesgo y/o un marcador de vulnerabilidad que puede precipitar el desarrollo de experiencias psicóticas clínicas y subclínicas (Hall y Degenhardt, 2008). Dada la compleja relación que se establece entre el riesgo de psicosis y el consumo de sustancias en los jóvenes y el impacto negativo que pueden generar en este periodo del desarrollo humano, es necesario realizar nuevos estudios que permitan comprender su asociación, con la finalidad de implementar medidas de promoción del bienestar emocional y prevención de trastornos mentales.

Hasta el momento, se han realizado escasos estudios que traten de analizar la relación entre el consumo de sustancias y el riesgo de psicosis (estimado mediante el constructo de esquizotipia) en muestras representativas de la población adolescente a nivel internacional, y más concretamente en España. Además, pocos trabajos previos han analizado dicha relación controlando el efecto de múltiples variables de confundido (p.ej., CI, nivel socio-económico) con instrumentos de evaluación específicamente diseñados para este sector de la población.

Dentro de este contexto de investigación, el objetivo principal de este trabajo fue explorar la relación entre el consumo de sustancias, concretamente alcohol, tabaco y cannabis, y los rasgos esquizotípicos en una muestra de adolescentes escolarizados españoles, controlando el efecto de múltiples variables de confundido. Se espera encontrar, de acuerdo a la literatura previa, que aquellos adolescentes que informen de mayor consumo de sustancias refieran mayores puntuaciones en los rasgos esquizotípicos de la personalidad.

\section{Método}

\section{Participantes}

Se planteó un diseño transversal descriptivo, tipo encuesta. Se realizó un muestreo aleatorio estratificado, por conglomerados, a nivel de aula de centro escolar, en una población aproximada de quince mil estudiantes seleccionados de la Comunidad Autónoma de La Rioja. Los alumnos pertenecían a diferentes centros escolares (públicos y privado-concertados) y Ciclos de Formación Profesional (básica, media y superior). Los estratos se crearon en función del centro escolar (público/privado) y la etapa escolar (ESO, Bachiller y Formación Profesional), en donde la probabilidad de extracción del aula del centro escolar venía dada en función del número de alumnos.

La muestra inicial estuvo formada por 1.881 estudiantes escolarizados, eliminando aquellos participantes que presentaron un puntaje alto en la Escala Oviedo de Infrecuencia de Respuesta (3 o más puntos) $(n=104)$, una edad mayor de $19(n=113)$ o no completaron la prueba $(n=76)$. Un total de 1.588 estudiantes, 739 hombres $(46,5 \%)$ y 849 mujeres $(53,5 \%)$, pertenecientes a 34 escuelas y 98 aulas participaron en el estudio. La edad promedio fue de 16,13 años (DT $=1,36)$, oscilando desde los 14 a los 19 años (14 años, $n=213$; 15 años, $n=337 ; 16$ años, $n=400 ; 17$ años, $n$ =382, 18 años, $n=180$; 19 años, $n=76$ ).

La distribución de la nacionalidad de los participantes fue la siguiente: $89,9 \%$ española, 3,7\% latinoamericana (Bolivia, Argentina, Colombia y Ecuador), 0,7\% portuguesa, $2,4 \%$ rumana, $1 \%$ marroquí, $0,7 \%$ pakistaní y $2 \%$ otras nacionalidades.

\section{Instrumentos}

Cuestionario Oviedo para la Evaluación de la Esquizotipia-revisado (ESQUIZO-Q) (Fonseca-Pedrero, Lemos, Muñiz, Paino y Villazón, 2010). Es un autoinforme compuesto por 62 ítems desarrollado para la evaluación de los rasgos esquizotípicos en población adolescente, que también puede ser utilizado con fines epidemiológicos. Los ítems del ESQUIZO-Q fueron seleccionados a partir de una revisión exhaustiva de la literatura de la personalidad esquizotípica. El formato de respuesta es tipo Likert de cinco categorías (1 "completamente en desacuerdo" y 5 "completamente de acuerdo"). El autoinforme se compone de un total de 10 subescalas derivadas empíricamente mediante análisis fac- 
torial exploratorio que, a su vez, se agrupan en tres dimensiones generales: Distorsión de la Realidad o Positiva (que incluye las subescalas de Ideación Referencial, Pensamiento Mágico, Experiencias Perceptivas Extrañas e Ideación Paranoide); Anhedonia (Anhedonia Física y Anhedonia Social); y Desorganización Social (Pensamiento y Lenguaje Extraños, Comportamiento Extraño, Ausencia de Amigos Íntimos y Ansiedad Social Excesiva).

Las propiedades psicométricas del ESQUIZO-Q han sido ampliamente analizadas en estudios previos (Fonseca-Pedrero, Lemos-Giráldez, Paino, Sierra-Baigrie, Santarén-Rosell y Muñiz, 2011; Fonseca-Pedrero, Paino, Lemos-Girádez y Muñiz, 2011).

Cuestionario de Consumo de Sustancias Modificado (Fonseca-Pedrero et al., 2020). El cuestionario de consumo de sustancias utilizado en la presente investigación es una modificación abreviada del Alcohol, Smoking and Substance Involvement Screening Test, de la Organización Mundial de la Salud (ASSIST v3.0) (WHO Assist Working Group, 2002). La ASSIST es una entrevista que se emplea como herramienta de cribado en la detección de consumidores de drogas. La ASSIST consta de diferentes ítems que evalúan, entre otros aspectos, la frecuencia de consumo de diferentes sustancias (alcohol, tabaco, cannabis, cocaína, etc.) en los tres meses anteriores a la realización del cuestionario.

En este estudio se utilizaron dos de las cuestiones de la ASSIST que se aplicaron en formato autoinforme. El ítem 1 preguntaba: "A lo largo de tu vida, ¿cuál de las siguientes sustancias has consumido alguna vez?” Los participantes debían responder en un formato de respuesta dicotómico Sí/ No para las siguientes sustancias: a) Tabaco (cigarrillos, cigarros habanos, tabaco de mascar, pipa, etc.); b) Bebidas alcohólicas (cerveza, vino, licores, destilados, etc.); c) Cannabis (marihuana, costo, hierba, hachís, etc.); y d) Otras (p.ej., cocaína, anfetaminas, inhalantes, alucinógenos, opiáceos, etc.). Por su parte, el ítem 2 preguntaba, en el caso de contestar afirmativamente a algunas de las sustancias del ítem 1 , sobre la frecuencia de consumo en los últimos tres meses $(0=$ "Nunca"; $1=$ "Una o dos veces"; $2=$ "Cada mes"; $3=$ "Cada semana"; $4=$ "A diario o casi a diario").

La ASSIST se encuentra traducida y validada al español (Soto-Brandt et al., 2014) y la versión modificada de la misma ha sido utilizada en adolescentes españoles (Fonseca-Pedrero et al., 2020).

Cuestionario de Capacidades y Dificultades-Autoinforme (Strengths and Difficulties Questionnaire-self-report version, SDQ) (Goodman, 1997). El SDQ es un instrumento de medida utilizado para la detección de dificultades conductuales y emocionales, así como para la valoración de capacidades en el ámbito prosocial (Fonseca-Pedrero et al., 2011). Ha sido utilizado también como herramienta de cribado y análisis epidemiológico del estado de salud mental en población infanto-juvenil (Ortuño-Sierra, Fonseca-Pedrero, Inchausti y Sastre i Riba, 2016).
El SDQ se compone de 25 ítems, en formato de respuesta Likert con tres opciones $(0=$ "No, nunca"; $1=$ "A veces"; $2=$ "Sí, siempre"). Los ítems se agrupan en cinco dimensiones (con cinco ítems cada una): Síntomas Emocionales, Problemas de Conducta, Dificultades de Hiperactividad, Problemas con Compañeros y Conducta Prosocial. Las primeras cuatro subescalas forman una puntuación Total de Dificultades. A mayor puntuación, mayor nivel de dificultades emocionales y comportamentales, excepto para la subescala de Conducta Prosocial, donde una menor puntuación es indicativa de un peor ajuste.

Las propiedades psicométricas del SDQ han sido analizadas en estudios previos nacionales (Ortuño-Sierra et al., 2016; Ortuño-Sierra, Fonseca-Pedrero, Paino, Sastre I Riba y Muñiz, 2015).

Test Matrices de Razonamiento de Penn (Penn Matrix Reasoning Test, PMRT) (Gur et al., 2012; Moore, Reise, Gur, Hakonarson y Gur, 2015). Esta es una tarea de la Batería Neurocognitiva Informatizada-Infantil de Pensilvania desarrollada para medir el razonamiento no verbal (usando problemas de razonamiento matricial como se usa en la Prueba de Matrices Progresivas de Raven). Esta tarea compuesta por 20 elementos se puede considerar como una estimación indirecta del cociente intelectual. Esta prueba ha sido utilizada en estudios previos con adolescentes españoles (Fonseca-Pedrero et al., 2020).

Family Affluence Scale-II (FAS-II) (Boyce, Torsheim, Currie y Zambon, 2006). La FAS-II permite una estimación indirecta del nivel socioeconómico mediante cuatro ítems con un sistema de respuesta tipo Likert. Ha mostrado su utilidad en población adolescente. Estudios internacionales previos han demostrado sus propiedades psicométricas adecuadas (Boyce, Torsheim, Currie y Zambon, 2006). La FAS-II, en sus versión española, ha sido utilizada en estudios previos (Fonseca-Pedrero et al., 2020).

Escala Oviedo de Infrecuencia de Respuesta (INF-OV) (Fonseca-Pedrero, Paino-Piñeiro, Lemos-Giráldez, Villazón-García y Muñiz, 2009). La INF-OV se ha desarrollado para detectar a aquellos participantes que han respondido de forma azarosa, pseudoazarosa o deshonesta a los instrumentos de medida administrados. La INF-OV es un instrumento de medida tipo autoinforme compuesto por 12 ítems en formato tipo Likert de cinco categorías en función del grado de adherencia (desde 1= "Completamente en desacuerdo" hasta $5=$ "Completamente de acuerdo"). Una vez dicotomizados los ítems, los alumnos que puntúan más de dos ítems de la INF-OV de forma incorrecta son eliminados del estudio. La INF-OV ha sido utilizada en trabajos previos (Fonseca-Pedrero et al., 2011; Fonseca-Pedrero et al., 2009).

\section{Procedimiento}

La investigación fue aprobada por la Dirección General de Educación del Gobierno de La Rioja y el Comité 
Ético de Investigación Clínica de La Rioja (CEICLAR). El contacto con los centros escolares se realizó por teléfono, e-mail o por correo postal. El primer contacto con el centro escolar se efectuaba con el director, el Jefe de Estudios, o con el Departamento de Orientación.

Con la finalidad de estandarizar el proceso de administración, se entregó a todos los investigadores un protocolo y unas normas que debían llevar a cabo antes, durante y después de la administración de los instrumentos de medida. La administración de los cuestionarios se realizó por ordenador y de forma colectiva en grupos de entre 10 y 30 participantes.

Se informó en todo momento de la confidencialidad de las respuestas, así como del carácter voluntario de la participación y no se dio gratificación alguna por la colaboración en el estudio. Dado que muchos de los participantes eran menores de edad, se solicitó un consentimiento paterno que autorizase la participación del adolescente en la investigación. Este estudio se enmarca dentro de un proyecto más amplio sobre la detección temprana de problemas de salud mental.

\section{Análisis de datos}

Con la finalidad de investigar los objetivos propuestos, se llevaron a cabo los siguientes análisis de datos.

En primer lugar, se analizó el porcentaje de participantes que informaban de consumo de sustancias, concretamente tabaco, alcohol y cannabis.

En segundo lugar, para examinar la relación entre el consumo de sustancias y las tres dimensiones de rasgos esquizotípicos (Positiva, Anhedonia y Desorganización Social) se llevaron a cabo diferentes Análisis Multivariados de la Covarianza (MANCOVA). Las dimensiones de esquizotipia fueron consideradas variables dependientes y el consumo de sustancias, (tabaco, alcohol y cannabis) factor fijo. Dentro de cada MANCOVA primero se analizó el efecto a nivel de prevalencia vital (haber consumido alguna vez en su vida) y luego a nivel de frecuencia. El género, la edad, el nivel socio-económico, el CI y/o los problemas emocionales y comportamentales fueron considerados covariables, dado que pueden afectar a la expresión de la relación entre los rasgos esquizotípicos y el consumo de sustancias. También, se consideró el consumo de sustancias como covariable, dependiendo del tipo de sustancia a estudiar. De este modo, al analizar el efecto del cannabis en las dimensiones de esquizotipia, se controló el efecto de consumo de alcohol y tabaco. Al analizar el efecto del tabaco en las dimensiones de esquizotipia, se controló el efecto de consumo de alcohol y cannabis. Al analizar el efecto del alcohol en las dimensiones de esquizotipia, se controló el efecto de consumo de cannabis y tabaco. El estadístico eta cuadrado parcial ( $\eta^{2}$ parcial) se utilizó para el cálculo del tamaño del efecto.

Los análisis de datos se llevaron a cabo con el programa estadístico SPSS v22 (IBM Corp Released, 2013).

\section{Resultados}

\section{Prevalencia de consumo de sustancias}

La prevalencia vital de consumo de sustancias (haber consumido alguna vez en su vida) fue del $40,6 \%$ para el tabaco, del 79,4\% para el alcohol y del $23,6 \%$ para el cannabis. El $15,7 \%$ de la muestra refirió beber alcohol cada semana. Además, el 2,9\% de los participantes presentó una frecuencia de consumo de cannabis mensual, mientras que un $2 \%$ presentó un patrón de consumo semanal. En la Tabla 1 se presentan las tasas de prevalencia de consumo de sustancias; se aportan además los datos de frecuencia de consumo de las 3 sustancias en los últimos 3 meses.

Tabla 1. Prevalencia y frecuencia de consumo de sustancias en la muestra total.

\begin{tabular}{|c|c|c|c|c|c|c|c|}
\hline & & \multicolumn{2}{|c|}{ Alcohol } & \multicolumn{2}{|c|}{ Tabaco } & \multicolumn{2}{|c|}{ Cannabis } \\
\hline & & $\mathbf{n}$ & $\%$ & $\mathbf{n}$ & $\%$ & $\mathbf{n}$ & $\%$ \\
\hline \multirow[t]{2}{*}{ Prevalencia } & No & 327 & 20,6 & 947 & 59,6 & 1211 & 76,3 \\
\hline & Sí & 1261 & 79,4 & 641 & 40,4 & 377 & 23,7 \\
\hline \multirow[t]{7}{*}{ Frecuencia } & Nunca (o) & 208 & 13,1 & 783 & 49,3 & 1047 & 65,9 \\
\hline & Una o dos veces (1) & 494 & 31,1 & 312 & 19,6 & 239 & 15,1 \\
\hline & Cada mes (2) & 478 & 30,1 & 77 & 4,8 & 46 & 2,9 \\
\hline & Cada semana (3) & 250 & 15,7 & 61 & 3,8 & 29 & 1,8 \\
\hline & A diario o casi a diario (4) & 14 & 0,9 & 163 & 10,3 & 29 & 1,8 \\
\hline & Total & 1444 & 90,9 & 1396 & 87,9 & 1390 & 87,5 \\
\hline & No respuesta & 144 & 9,1 & 192 & 12,1 & 198 & 12,5 \\
\hline
\end{tabular}

Nota. Prevalencia: el participante indica si ha consumido o no la sustancias alguna vez en su vida. 


\section{Relación entre consumo de alcohol y rasgos esquizotípicos}

En primer lugar, se analizó la relación entre la prevalencia vital de consumo de alcohol y las puntuaciones del ESQUIZO-Qy, a continuación, entre frecuencia de consumo y las puntuaciones del ESQUIZO-Q. Se controló el efecto del género, la edad, el CI, el nivel socio-económico, la psicopatología y el consumo de cannabis y tabaco.

En función de la prevalencia vital de consumo de alcohol, el MANCOVA no reveló la existencia de diferencias estadísticamente significativas $\left(\lambda=0,998 ; F_{(3,1577)}=1,110, p\right.$ $=0,334)$. Los consumidores de alcohol, comparados con los no consumidores, no informaron de mayores puntuaciones medias en ninguna de las dimensiones del ESQUI-
ZO-Q. Las puntuaciones medias para los dos grupos se recogen en la Tabla 2.

En función de la frecuencia de uso de alcohol (en los últimos 3 meses), el MANCOVA reveló la existencia de diferencias estadísticamente significativas $\left(\lambda=0,985 ; F_{112}\right.$ $\left.{ }_{3783)}=1,842, p=0,037\right)$. Aquellos que refirieron una mayor frecuencia de consumo de alcohol, comparados con los no consumidores, informaron de mayores puntuaciones medias en la dimensión Desorganización Social. Concretamente, se encontraron diferencias estadísticamente significativas entre el grupo 1 ("una o dos veces") y 3 ("cada semana"). Las puntuaciones medias para los grupos se recogen en la Tabla 3 .

Tabla 2. Relación entre consumo de alcohol (prevalencia vital) y rasgos esquizotípicos.

\begin{tabular}{|c|c|c|c|c|c|c|c|}
\hline \multirow[b]{2}{*}{ Dimensión } & \multicolumn{2}{|c|}{ No consumo } & \multicolumn{2}{|c|}{ Consumo } & \multirow[b]{2}{*}{$F$} & \multirow[b]{2}{*}{$p$} & \multirow[b]{2}{*}{$\eta^{2}$ parcial } \\
\hline & $M$ & $D T$ & $M$ & $D T$ & & & \\
\hline Positiva & 33,51 & 11,69 & 34,65 & 11,91 & 0,4 & 0,527 & 0 \\
\hline Negativa & 34,52 & 7,33 & 33,44 & 6,54 & 1,99 & 0,159 & 0,001 \\
\hline Desorganización & 57,47 & 15,81 & 58,85 & 15,23 & 0,554 & 0,457 & 0 \\
\hline
\end{tabular}

Tabla 3. Relación entre consumo de alcohol (frecuencia) y rasgos esquizotípicos.

\begin{tabular}{|c|c|c|c|c|c|c|c|c|c|c|c|c|c|}
\hline \multirow[b]{2}{*}{ Dimensión } & \multicolumn{2}{|c|}{$\mathbf{0}$} & \multicolumn{2}{|c|}{1} & \multicolumn{2}{|c|}{2} & \multicolumn{2}{|c|}{3} & \multicolumn{2}{|c|}{4} & \multirow[b]{2}{*}{$F$} & \multirow[b]{2}{*}{$p$} & \multirow[b]{2}{*}{$\eta^{2}$ parcial } \\
\hline & $M$ & $D T$ & $M$ & $D T$ & $M$ & $D T$ & $M$ & $D T$ & $M$ & $D T$ & & & \\
\hline Positiva & 33,80 & 11,19 & 34,50 & 11,90 & 34,50 & 11,28 & 35,06 & 12,19 & 40,50 & 24,42 & 0,718 & 0,579 & 0,002 \\
\hline Negativa & 34,02 & 7,26 & 33,59 & 6,75 & 32,92 & 6,24 & 33,90 & 6,54 & 34,29 & 7,34 & 0,494 & 0,74 & 0,001 \\
\hline Desorganización & 57,38 & 15,73 & 59,92 & 15,31 & 58,26 & 14,71 & 57,87 & 14,87 & 57,50 & 26,39 & 2,676 & 0,031 & 0,007 \\
\hline
\end{tabular}

Nota . Nunca $=0 ;$ Una o dos veces $=1 ;$ Cada mes $=2 ;$ Cada semana $=3 ;$ A diario o casi a diario $=4$.

\section{Relación entre consumo de tabaco y rasgos esquizotípicos}

El MANCOVA que se utilizó para analizar la relación entre el consumo o no de tabaco (prevalencia vital) y las puntuaciones del ESQUIZO-Q, controlando el efecto del género, la edad, el CI, el nivel socio-económico, la psicopatología y el consumo de cannabis y alcohol, reveló la existencia de diferencias estadísticamente significativas $(\lambda$ $\left.=0,991 ; F_{(3,1577)}=4,995, p=0,002\right)$, concretamente en las dimensiones Anhedonia y Desorganización Social. Los consumidores de tabaco, comparados con los no consumidores, informaron de mayores puntuaciones medias en estas dos dimensiones del ESQUIZO-Q. Las puntuaciones medias para los dos grupos se presentan en la Tabla 4.

A continuación, se realizó un nuevo MANCOVA considerando la variable frecuencia de uso de tabaco como fac- tor fijo, las dimensiones del ESQUIZO-Q como variables dependientes, y controlando el efecto de las mismas covariables. En este caso, el MANCOVA reveló la existencia de diferencias estadísticamente significativas $\left(\lambda=0,973 ; F_{(12}\right.$, $\left.{ }_{3656)}=3,143, p<0,001\right)$. Los consumidores de tabaco diferenciados en función de su frecuencia, comparados con los no consumidores, informaron de mayores puntuaciones medias en las dimensiones Anhedonia y Desorganización Social del ESQUIZO-Q. Concretamente se encontraron diferencias estadísticamente significativas entre el grupo 0 y 1 en Anhedonia, y entre los grupos 0-1, 0-4 y 2-4 ( siendo Nunca $=0$; Una o dos veces $=1$; Cada mes $=2$; Cada semana $=3$; A diario o casi a diario $=4$ ) en el factor esquizotípico de Desorganización. Las puntuaciones medias para los grupos de frecuencia de uso de tabaco, se recogen en la Tabla 5 . 
Tabla 4. Relación entre consumo de tabaco (prevalencia vital) y rasgos esquizotípicos.

\begin{tabular}{|c|c|c|c|c|c|c|c|}
\hline \multirow[b]{2}{*}{ Dimensión } & \multicolumn{2}{|c|}{ No consumo } & \multicolumn{2}{|c|}{ Consumo } & \multirow[b]{2}{*}{$F$} & \multirow[b]{2}{*}{$p$} & \multirow[b]{2}{*}{$\eta^{2}$ parcial } \\
\hline & $M$ & $D T$ & $M$ & $D T$ & & & \\
\hline Positiva & 33,81 & 11,54 & 35,31 & 12,30 & 1,132 & 0,287 & 0,001 \\
\hline Negativa & 33,95 & 6,87 & 33,24 & 6,47 & 5,707 & 0,017 & 0,004 \\
\hline Desorganización & 58,14 & 15,26 & 59,20 & 15,49 & 11,93 & 0,001 & 0,007 \\
\hline
\end{tabular}

Tabla 5. Relación entre consumo de tabaco (frecuencia) y rasgos esquizotípicos.

\begin{tabular}{|c|c|c|c|c|c|c|c|c|c|c|c|c|c|}
\hline \multirow[b]{2}{*}{ Dimensión } & \multicolumn{2}{|c|}{ o } & \multicolumn{2}{|c|}{1} & \multicolumn{2}{|c|}{2} & \multicolumn{2}{|c|}{3} & \multicolumn{2}{|c|}{4} & \multirow[b]{2}{*}{$F$} & \multirow[b]{2}{*}{$p$} & \multirow[b]{2}{*}{$\eta^{2}$ parcial } \\
\hline & $M$ & $D T$ & $M$ & $D T$ & $M$ & $D T$ & $M$ & $D T$ & $M$ & $D T$ & & & \\
\hline Positiva & 34,14 & 11,68 & 34,40 & 11,46 & 36,32 & 12,14 & 36,84 & 11,02 & 35,61 & 13,49 & 1,638 & 0,162 & 0,005 \\
\hline Negativa & 33,74 & 6,78 & 32,66 & 6,33 & 32,16 & 6,12 & 33,77 & 6,95 & 34,24 & 6,53 & 2,888 & 0,021 & 0,008 \\
\hline Desorganización & 58,31 & 15,14 & 58,63 & 14,52 & 60,06 & 13,78 & 60,97 & 16,76 & 58,86 & 16,87 & 6,444 & $<0,001$ & 0,018 \\
\hline
\end{tabular}

Nota . Nunca $=0 ;$ Una o dos veces $=1 ;$ Cada mes $=2 ;$ Cada semana $=3 ;$ A diario o casi a diario $=4$.

\section{Relación entre consumo de cannabis y rasgos esquizotípicos}

El MANCOVA sobre la relación entre prevalencia vital de consumo de cannabis y las dimensiones de esquizotipia, controlando el efecto de las covariables género, edad, CI, nivel socio-económico, psicopatología y consumo de alcohol y tabaco, no reveló la existencia de diferencias estadísticamente significativas $\left(\lambda=0,998 ; F_{(3,1577)}=1,146, p=0,329\right)$. Los consumidores de cannabis, comparados con los no consumidores, no informaron de mayores puntuaciones medias en ninguna de las dimensiones del ESQUIZO-Q. Los resultados se presentan en la Tabla 6 .
En función de la frecuencia de consumo, el MANCOVA realizado, considerando la variable frecuencia de uso de cannabis como factor fijo, las puntuaciones del ESQUIZO-Q como variables dependientes, y controlando el efecto de las mismas covariables, tampoco reveló la existencia de diferencias estadísticamente significativas $(\lambda=0,991$; $\left.F_{(12,3640)}=1,014, p=0,433\right)$. Las puntuaciones medias para los distintos grupos de frecuencia de consumo de cannabis se muestran en la Tabla 7.

Tabla 6. Relación entre consumo de cannabis (prevalencia vital) y rasgos esquizotípicos.

\begin{tabular}{|c|c|c|c|c|c|c|c|}
\hline \multirow[b]{2}{*}{ Dimensión } & \multicolumn{2}{|c|}{ No consumo } & \multicolumn{2}{|c|}{ Consumo } & \multirow[b]{2}{*}{$F$} & \multirow[b]{2}{*}{$p$} & \multirow[b]{2}{*}{$\eta^{2}$ parcial } \\
\hline & $M$ & $D T$ & $M$ & $D T$ & & & \\
\hline Positiva & 33,79 & 11,43 & 36,43 & 13,01 & 1,743 & 0,187 & 0,001 \\
\hline Negativa & 33,57 & 6,73 & 33,95 & 6,70 & 1,377 & 0,241 & 0,001 \\
\hline Desorganización & 58,03 & 15,16 & 60,29 & 15,88 & 0,042 & 0,838 & 0 \\
\hline
\end{tabular}

Tabla 7. Relación entre consumo de cannabis (frecuencia) y rasgos esquizotípicos.

\begin{tabular}{|c|c|c|c|c|c|c|c|c|c|c|c|c|c|}
\hline \multirow[b]{2}{*}{ Dimensión } & \multicolumn{2}{|c|}{ o } & \multicolumn{2}{|c|}{1} & \multicolumn{2}{|c|}{2} & \multicolumn{2}{|c|}{3} & \multicolumn{2}{|c|}{4} & \multirow[b]{2}{*}{$F$} & \multirow[b]{2}{*}{$p$} & \multirow[b]{2}{*}{$\eta^{2}$ parcia } \\
\hline & $M$ & $D T$ & $M$ & $D T$ & $M$ & $D T$ & $M$ & $D T$ & $M$ & $D T$ & & & \\
\hline Positiva & 33,99 & 11,41 & 36,04 & 12,77 & 37,22 & 11,50 & 35,31 & 12,04 & 38,55 & 17,82 & 0,788 & 0,533 & 0,002 \\
\hline Negativa & 33,31 & 6,66 & 33,31 & 6,38 & 35,09 & 8,28 & 35,31 & 5,50 & 35,14 & 6,96 & 0,43 & 0,787 & 0,001 \\
\hline Desorganización & 58,07 & 14,96 & 59,67 & 15,50 & 62,70 & 15,54 & 59,86 & 16,36 & 59,76 & 20,12 & 1,266 & 0,0281 & 0,004 \\
\hline
\end{tabular}

Nota. Nunca $=0 ;$ Una o dos veces $=1 ;$ Cada mes $=2 ;$ Cada semana $=3 ;$ A diario o casi a diario $=4$. 


\section{Discusión}

El objetivo principal de este estudio fue analizar la relación entre el consumo de sustancias (tabaco, alcohol y cannabis) y el riesgo de psicosis, estimado mediante rasgos esquizotípicos, en una muestra representativa de adolescentes españoles escolarizados.

En primer lugar, se analizaron las tasas de prevalencia vital de consumo de alcohol, tabaco y cannabis. Los resultados encontrados muestran una prevalencia vital para el tabaco del $40,4 \%$, del 79,4\% para el alcohol y del $23,7 \%$ para el cannabis. Estos resultados son similares a los obtenidos en la Encuesta sobre Uso de Drogas en Enseñanzas Secundarias en España ESTUDES 16-17 (34,7\% para el tabaco, $76,9 \%$ alcohol y $31,1 \%$ cannabis). De la muestra, un $10,3 \%$ de los adolescentes informaron que fumaban tabaco a diario o casi a diario; según el Observatorio de Drogas en España, los jóvenes que manifestaron un consumo diario de tabaco representan un 8,8\% (Moreno, 2018). El 30,1\% consumía alcohol cada mes; dato similar a los estudiantes de secundaria (ESTUDES 16-17) donde 3 de cada 4 jóvenes admiten haber bebido alcohol en los últimos 30 días. Por último, un 2,9\% informó que fumaba cannabis mensualmente, cifra que contrasta con los estudiantes de Enseñanza Secundaria de 14 a 18 años de (ESTUDES 16-17) que confirman un consumo de cannabis de un 18,3\% en los últimos 30 días. Datos de un reciente estudio revelan que, a día de hoy, es ya mayor el porcentaje de adolescentes que consumen tabaco y cannabis que únicamente tabaco $(12,7 \%$ vs $10,5 \%)$ y que ello no sólo implica una mayor probabilidad de consumir otras sustancias ilegales, sino también de desarrollar un patrón consumo de alcohol de riesgo (Rial et al., 2018). Según diversos estudios, los jóvenes no son conscientes de las conductas de riesgo que conlleva el consumo de drogas; por la baja percepción de riesgo, consideran como inofensivo el consumo esporádico, lo cual les hace más vulnerables (Barrett y Bradley, 2016; López-Quintero y Neumark, 2010; Rowe, Santos, Behar y Coffin, 2016).

En segundo lugar, se analizaron las dimensiones de esquizotipia (Positiva, Anhedonia y Desorganización) en función del consumo -a nivel de prevalencia vital y a nivel de frecuencia- controlando el efecto de las covariables (género, edad, CI, nivel socio-económico, psicopatología y consumo de otras sustancias). Los consumidores de alcohol comparados con los no consumidores, informaron de puntuaciones más altas en la dimensión de Desorganización Social. Hallazgos que coinciden con una revisión actual sobre los efectos del consumo intensivo de alcohol en jóvenes, que asocian dicho consumo a déficits neurocognitivos (disminuye la atención sostenida, la memoria de trabajo visoespacial; déficit en la toma de decisiones, flexibilidad cognitiva y funciones ejecutivas) (López-Caneda et al., 2014). Las dimensiones Positiva y Anhedonia no presentaron diferencias estadísticamente significativas cuando se controlaron los efectos de dichas covariables.
En la misma línea que nuestra investigación, Auther et al. (2012) hallaron que los adolescentes con un inicio temprano (17 años o menos) en el consumo de alcohol fueron significativamente más propensos a mostrar experiencias esquizotípicas. Sin embargo, el patrón de asociación entre el uso de alcohol, trastorno de dependencia y experiencias esquizotípicas fue menos consistente cuando se ajustó para la presencia de otros trastornos por consumo de sustancias, a pesar de que los síntomas más severos también estaban asociados con niveles más altos de consumo de sustancias.

Los consumidores de tabaco informaron de mayores puntuaciones en las dimensiones de Anhedonia y Desorganización Social. Los resultados del presente trabajo reflejan que, tanto la presencia de consumo (sí/no), como la frecuencia de consumo, se asocian con puntuaciones más altas en los rasgos negativos y de desorganización social. La anhedonia se asocia al hábito de fumar para el alivio de estos efectos (Leventhal et al., 2013) y es considerada como un marcador de riesgo a la psicosis (Docherty y Sponheim, 2014; Meehl, 1962). Varios estudios prospectivos vinculan el tabaquismo con síntomas psicóticos (Gurillo et al., 2015; Munafò, Larsson Lönn, Sundquist, Sundquist y Kendler, 2016; Riala, Hakko, Isohanni, Pouta y Räsänen, 2005; Zammit et al., 2003). Por ejemplo, Gurillo et al. (2015) destacan que el consumo diario de tabaco se asocia con un mayor riesgo de psicosis en los estudios de casos y controles; indicando asimismo que el hecho de fumar se relaciona con una edad más temprana en el inicio del trastorno psicótico. En el mismo sentido, Weiser et al. (2004) afirmaron que la tasa de consumo de cigarrillos en adolecentes varones se asoció significativamente con el riesgo de esquizofrenia. No obstante, la posibilidad de un vínculo causal entre el consumo de tabaco y la psicosis merece un examen más detenido.

Con respecto a la influencia del consumo de cannabis, no se hallaron diferencias estadísticamente significativas, ni en función de prevalencia vital de consumo, ni de frecuencia de consumo, en las dimensiones de esquizotipia, cuando se controló el efecto de variables de confundido. Los resultados encontrados no revelan una asociación estadísticamente significativa, una vez ajustados los valores de confusión, sugiriendo esto la posible existencia de factores subyacentes que expliquen en mayor medida la asociación. Sin embargo, un estudio en España encontró que, los pacientes que presentaban un primer episodio de psicosis (edad promedio 15,5 años), tenían una tasa más alta de síntomas positivos y menos síntomas negativos si eran consumidores de cannabis, en comparación con los que no consumían cannabis (Baeza et al., 2009). La asociación entre el consumo de cannabis y psicosis ha sido analizada en una gran cantidad de estudios previos (Fergusson, Horwood y Ridder, 2005; Fonseca-Pedrero, Ortuño-Sierra, Paino y Muñiz, 2016; Gage, Hickman y Zammit, 2016; Hides et al., 2009; Kelleher et al., 2012; Marconi et al., 2016; Moore et al., 2007). En general, las investigaciones en el 
campo de la esquizotipia indican que las relaciones que se establecen con el consumo de cannabis son complejas y bidireccionales, donde diferentes variables pueden estar jugando un rol mediador (Fonseca-Pedrero et al., 2020). Por ejemplo, Schubart et al. (2011), analizaron la asociación entre la edad de inicio y nivel de consumo de cannabis y experiencias psicóticas en tres dimensiones de los síntomas (positiva, negativa y depresiva) en una muestra de población de Países Bajos de más de 17.500 participantes, con una edad promedio de 21 años. En su estudio, encontraron que la edad de inicio de consumo de cannabis estaba fuertemente asociada con experiencias psicóticas actuales y que el nivel de consumo de cannabis estaba igualmente relacionado con síntomas positivos, negativos y depresivos.

Existe una gran cantidad de literatura que vincula el consumo de sustancias con la psicosis, si bien es difícil establecer la causalidad a partir de estas investigaciones. Como afirman Minozzi et al. (2009), la causalidad inversa y la confusión residual no pueden ser excluidas. La interacción con otros factores ambientales y genéticos es difícil de determinar. Esto no debería alterar el mensaje de salud pública de que el cannabis puede ser dañino y que la dependencia del cannabis debería evitarse (Gage et al., 2016). De hecho, tal vez, una línea de actuación interesante en la prevención de la psicosis, más que reducir el riesgo o la vulnerabilidad latente en sí misma, sería eliminar o reducir factores de riesgo relevantes que se encuentran íntimamente relacionados con su etiología como, por ejemplo, el consumo de cannabis o las experiencias de trauma (Radua et al., 2018).

A la hora de interpretar los resultados obtenidos en el presente trabajo, resulta necesario mencionar algunas limitaciones. Primero, las conclusiones se encuentran mediatizadas por los instrumentos de medida utilizados, de tipo autoinforme, con las correspondientes acotaciones de este tipo de herramientas (posible falta de comprensión e interpretación de los ítems o los sesgos de respuesta). El sistema multi-informante podría tener una especial relevancia en la evaluación de las variables de estudio. Segundo, la muestra pertenece a una Comunidad Autónoma española (La Rioja) aspecto que, aunque se haya realizado un muestreo aleatorio estratificado por conglomerados, limita parcialmente la generalización de los resultados a todo el territorio español. Tercero, la naturaleza transversal del estudio limita el establecimiento de relaciones causa-efecto. Cuarto, no se recogió información de la posible morbilidad psiquiátrica, ni de los participantes, ni de los familiares cercanos, la cual podría afectar a los resultados encontrados en el trabajo.

En conclusión, los rasgos esquizotípicos se encontraron asociados al consumo de tabaco y alcohol, pero no al cannabis. Este estudio pretende arrojar luz sobre la relación entre experiencias de consumo y rasgos esquizotípicos en la adolescencia. Es preciso identificar los mecanismos que subyacen a esta asociación en grupos vulnerables de jóve- nes de riesgo con el objetivo de mejorar las estrategias de prevención. Futuros estudios podrían seguir analizando el papel de los factores de riesgo y de protección que se relacionan con la transición a diferentes problemas psicológicos mediante estudios longitudinales y de interacción gen-persona-ambiente. Del mismo modo, es sumamente interesante incorporar nuevas tecnologías de evaluación y medición como el método de muestreo de experiencias que permita un análisis de la conducta humana más ecológico, contextual, etiológico, personalizado y de precisión.

\section{Agradecimientos}

Esta investigación ha sido financiada por el Ministerio de Ciencia e Innovación de España (MICINN) (referencia PSI2014-56114-P), por el Instituto Carlos III, Centro de Investigación Biomédica en Red de Salud Mental (CIBERSAM), por la Convocatoria 2015 de "Ayudas Fundación BBVA a Investigadores y Creadores Culturales”, por las "Ayudas Fundación BBVA a Equipos de Investigación Científica 2017”, por el Ministerio de Economía y Competitividad (MINECO) (referencia PSI 2016-79524-R) y cofinanciado con fondos FEDER en el PO FEDER de La Rioja 2014-2020 (SRS 6FRSABC026).

\section{Conflicto de intereses}

Los autores declaran no presentar conflictos de intereses.

\section{Referencias}

Abajobir, A. A., Kisely, S., Scott, J. G., Williams, G., Clavarino, A., Strathearn, L. y Najman, J. M. (2017). Childhood maltreatment and young adulthood hallucinations, delusional experiences, and psychosis: a longitudinal study. Schizophrenia Bulletin, 43, 1045-1055. doi:10.1093/ schbul/sbw175.

Arseneault, L., Cannon, M., Fisher, H. L., Polanczyk, G., Moffitt, T. E. y Caspi, A. (2011). Childhood trauma and children's emerging psychotic symptoms: a genetically sensitive longitudinal cohort study. American Journal of Psychiatry, 168, 65-72. doi:10.1176/appi. ajp.2010.10040567.

Auther, A. M., McLaughlin, D., Carrión, R. E., Nagachandran, P., Correll, C. U. y Cornblatt, B. A. (2012). Prospective study of cannabis use in adolescents at clinical high risk for psychosis: impact on conversion to psychosis and functional outcome. Psychological Medicine, 42, 2485-2497. doi:10.1017/S0033291712000803.

Baeza, I., Graell, M., Moreno, D., Castro-Fornieles, J., Parellada, M., González-Pinto, A., ... Arango, C. (2009). Can- 
nabis use in children and adolescents with first episode psychosis: influence on psychopathology and short-term outcome (CAFEPS study). Schizophrenia Research, 113, 129-137. doi:10.1016/j.schres.2009.04.005.

Barkus, E. (2008). Personality and cannabis use. Advances in Schizophrenia and Clinical Psychiatry, 3, 84-89.

Barrantes-Vidal, N., Grant, P. y Kwapil, T. R. (2015). The role of schizotypy in the study of the etiology of schizophrenia spectrum disorders. Schizophrenia Bulletin, 41, 408-416. doi:10.1093/schbul/sbu191.

Barrett, P. y Bradley, C. (2016). Attitudes and perceived risk of cannabis use in Irish adolescents. Irish Journal of Medical Science, 185, 643-647. doi:10.1007/s11845-015-1325-2.

Boyce, W., Torsheim, T., Currie, C. y Zambon, A. (2006). The Family Affluence Scale as a measure of national wealth: validation of an adolescent self-report measure. Social Indicators Research, 78, 473-487. doi:10.1007/ s11205-005-1607-6.

Buchy, L., Perkins, D., Woods, S. W., Liu, L. y Addington, J. (2014). Impact of substance use on conversion to psychosis in youth at clinical high risk of psychosis. Schizophrenia Research, 156, 277-280. doi:10.1016/j. schres.2014.04.021.

Casajuana Kögel, C., López-Pelayo, H., Balcells-Olivero, M. M., Colom, J. y Gual, A. (2018). Psychoactive constituents of cannabis and their clinical implications: a systematic review constituyentes psicoactivos del cannabis y sus implicaciones clínicas: una revisión sistemática. Adicciones, 30, 140-151. doi:10.20882/adicciones.858.

Cohen, A. S., Buckner, J. D., Najolia, G. M. y Stewart, D. W. (2011). Cannabis and psychometrically-defined schizotypy: use, problems and treatment considerations. Journal of Psychiatric Research, 45, 548-554. doi:10.1016/j. jpsychires.2010.08.013.

Cohn, A., Johnson, A., Ehlke, S. y Villanti, A. C. (2016). Characterizing substance use and mental health profiles of cigar, blunt, and non-blunt marijuana users from the National Survey of Drug Use and Health. Drug and Alcohol Dependence, 160, 105-111. doi:10.1016/j.drugalcdep.2015.12.017.

Debbané, M., Eliez, S., Badoud, D., Conus, P., Fluckiger, R. y Schultze-Lutter, F. (2015). Developing psychosis and its risk states through the lens of schizotypy. Schizophrenia Bulletin, 41, 396-407. doi:10.1093/schbul/sbu176.

Ericson, M., Tuvblad, C., Raine, A., Young-Wolff, K. y Baker, L. A. (2011). Heritability and longitudinal stability of schizotypal traits during adolescence. Behavior Genetics, 41, 499-511. doi:10.1007/s10519-010-9401-x.

Fergusson, D. M., Horwood, L. J. y Ridder, E. M. (2005). Tests of causal linkage between cannabis use and psychotic symptoms. Addiction, 100, 354-366. doi:10.1111/ j.1360-0443.2005.001001.x.

Fonseca-Pedrero, E., Lemos, S., Muñiz, J., Paino, M. y Villazón, U. (2010). ESQUIZO-Q. Cuestionario Oviedo para la Evaluación de la Esquizotipia. Madrid: TEA Ediciones.

Fonseca-Pedrero, E. (coord). (2018). Evaluación de los trastornos del espectro psicótico. Madrid: Pirámide.

Fonseca-Pedrero, E. y Debbané, M. (2017). Schizotypal traits and psychotic-like experiences during adolescence: an update. Psicothema, 29, 5-17. doi:10.7334/psicothema2016.209.

Fonseca-Pedrero, E. e Inchausti, F. (2018). Update on the prevention of psychotic spectrum disorders. $P a$ peles del Psicologo, 39, 127-139. doi:10.23923/pap.psicol2018.2860.

Fonseca-Pedrero, E., Lucas-Molina, B., Pérez-Albéniz, A., Inchausti, F. y Ortuño-Sierra, J. (2020). Psychotic-like experiences and cannabis use in adolescents from the general population. Adicciones, 32, 41-51. doi:10.20882/ adicciones.1149.

Fonseca-Pedrero, E., Ortuño-Sierra, J., Paino, M. y Muñiz, J. (2016). Psychotic-like experiences and substance use in college students. Adicciones, 28, 144-153. doi:10.20882/ adicciones.781.

Fonseca-Pedrero, E., Paino-Piñeiro, M., Lemos-Giráldez, S., Villazón-García, U. y Muñiz, J. (2009). Validation of the Schizotypal Personality Questionnaire-Brief form in adolescents. Schizophrenia Research, 111, 53-60. doi:10.1016/j.schres.2009.03.006.

Fonseca-Pedrero, E., Paino, M., Lemos-Giráldez, S. y Muñiz, J. (2011a). Prevalencia de la sintomatología emocional y comportamental en adolescentes Españoles a través del Strengths and Difficulties Questionnaire (SDQ). Revista de Psicopatologia y Psicologia Clinica, 16, 15-25. doi:10.5944/rppc.vol.16.num.1.2011.10348.

Fonseca-Pedrero, E., Paino, M., Lemos-Giráldez, S. y Muñiz, J. (2011b). Schizotypal traits and depressive symptoms in nonclinical adolescents. Comprehensive Psychiatry, 52, 293-300. doi:10.1016/j.comppsych.2010.07.001.

Fonseca-Pedrero, E., Paino, M., Lemos-Giráldez, S., Sierra-Baigrie, S., Ordóñez-Camblor, N. y Muñiz, J. (2011). Early psychopathological features in Spanish adolescents. Psicothema, 23, 87-93.

Fumero, A., Marrero, R. J. y Fonseca-Pedrero, E. (2018). Well-being in schizotypy: the effect of subclinical psychotic experiences. Psicothema, 30, 177-182. doi:10.7334/ psicothema2017.100.

Fumero A., Santamaría, C. y Navarrete, G. (2009). Predisposición al consumo de alcohol y drogas en personas vulnerables a la esquizofrenia. Revista de Neurologia, 49, 8-12. doi:10.33588/rn.4901.2008672.

Gage, S. H., Hickman, M. y Zammit, S. (2016). Association between cannabis and psychosis: epidemiologic evidence. Biological Psychiatry, 79, 549-556. doi:10.1016/j. biopsych.2015.08.001.

García Montes, J. M., Zaldívar Basurto, F., Moreno Montoya, M. y Flores Cubos, P. (2013). Relaciones entre el 
consumo de drogas y variables de riesgo psicopatológicas en jóvenes universitarios. Psicothema, 25, 433-439. doi:10.7334/psicothema2013.20.

Gonzalvo, B., Barral, C. y Roncero, O. E. C. (2011). Comorbilidad psiquiátrica en adolescentes consumidores de cannabis. Trastornos Adictivos, 13, 109-112. doi:10.1016/ S1575-0973(11)70023-X.

Goodman, R. (1997). The Strengths and Difficulties Questionnaire: a research note. Journal of Child Psychology and Psychiatry, 38, 581-6. doi:10.1111/j.1469-7610.1997. tb01545.x.

Gur, R. C., Richard, J., Calkins, M. E., Chiavacci, R., Hansen, J. A., Bilker, W. B., ... Gur, R. E. (2012). Age group and sex differences in performance on a computerized neurocognitive battery in children age 8-21. Neuropsychology, 26, 251-265. doi:10.1037/a0026712.

Gurillo, P., Jauhar, S., Murray, R. M. y MacCabe, J. H. (2015). Does tobacco use cause psychosis? Systematic review and meta-analysis. Lancet Psychiatry, 2, 718-725. doi:10.1016/S2215-0366(15)00152-2.

Hall, W. y Degenhardt, L. (2008). Cannabis use and the risk of developing a psychotic disorder. World Psychiatry, 7, 68-71.

Hides, L., Lubman, D. I., Buckby, J., Yuen, H. P., Cosgrave, E., Baker, K. y Yung, A. R. (2009). The association between early cannabis use and psychotic-like experiences in a community adolescent sample. Schizophrenia Research, 112, 130-135. doi:10.1016/j.schres.2009.04.001.

IBM Corp Released. (2013). IBM SPSS Statistics for Windows, Version 22.0. Armonk, NY: IBM Corp.

Kelleher, I., Connor, D. y Clarke, M. C. (2012). Prevalence of psychotic symptoms in childhood and adolescence: a systematic review and meta- analysis of population-based studies. Psychological Medicine, 42, 1857-1863. doi:10.1017/S0033291711002960.

Kraan, T., Velthorst, E., Koenders, L., Zwaart, K., Ising, H. K., van den Berg, D., ... Van der Gaag, M. (2016). Cannabis use and transition to psychosis in individuals at ultra-high risk: review and meta-analysis. Psychological Medicine, 46, 673-681. doi:10.1017/S0033291715002329.

Large, M., Sharma, S., Compton, M. T., Slade, T. y Nielssen, O. (2011). Cannabis use and earlier onset of psychosis: a systematic meta-analysis. Archives of General Psychiatry, 68, 555-561. doi:10.1001/archgenpsychiatry.2011.5.

Leventhal, A. M., Greenberg, J. B., Trujillo, M. A., Ameringer, K. J., Lisha, N. E., Pang, R. D. y Monterosso, J. (2013). Positive and negative affect as predictors of urge to smoke: temporal factors and mediational pathways. Psychology of Addictive Behaviors, 27, 262-267. doi:10.1037/a0031579.

Linscott, R. J. y Van Os, J. (2013). An updated and conservative systematic review and meta-analysis of epidemiological evidence on psychotic experiences in children and adults: on the pathway from proneness to persistence to dimensional expression across mental disor- ders. Psychological Medicine, 43, 1133-1149. doi:10.1017/ S0033291712001626.

López-Caneda, E., Mota, N., Crego, A., Velásquez, T., Corral, M., Holguín, S. R. y Cadaveira, F. (2014). Anomalías neurocognitivas asociadas al consumo intensivo de alcohol (binge drinking) en jóvenes y adolescentes: Una revisión. Adicciones, 26, 334-359. doi:10.20882/adicciones.39.

López-Quintero, C. y Neumark, Y. (2010). Effects of risk perception of marijuana use on marijuana use and intentions to use among adolescents in Bogotá, Colombia. Drug and Alcohol Dependence, 109, 65-72. doi:10.1016/j. drugalcdep.2009.12.011.

Malone, D. T., Hill, M. N. y Rubino, T. (2010). Adolescent cannabis use and psychosis: epidemiology and neurodevelopmental models. British Journal of Pharmacology, 160, 511-522. doi:10.1111/j.1476-5381.2010.00721.x.

Marconi, A., Di Forti, M., Lewis, C. M., Murray, R. M. y Vassos, E. (2016). Meta-analysis of the association between the level of cannabis use and risk of psychosis. Schizophrenia Bulletin, 42, 1262-1269. doi:10.1093/schbul/sbw003.

Maturana, A. (2011). Consumo de alcohol y drogas en adolescentes. Revista Médica Clínica Las Condes, 22, 98-109. doi:10.1016/S0716-8640(11)70397-2.

Meehl, P. E. (1962). Schizotaxia, schizotypy, schizophrenia. American Psychologist, 17, 827-838. doi:10.1037/ h0041029.

Minozzi, S., Davoli, M., Bargagli, A. M., Amato, L., Vecchi, S. y Perucci, C. A. (2009). An overview of systematic reviews on cannabis and psychosis: discussing apparently conflicting results. Drug and Alcohol Review, 29, 304-317. doi:10.1111/j.1465-3362.2009.00132.x.

Moore, T. H. M., Zammit, S., Lingford-Hughes, A., Barnes, T. R. E., Jones, P. B., Burke, M. y Lewis, G. (2007). Cannabis use and risk of psychotic or affective mental health outcomes: a systematic review. Lancet, 370, 319-28. doi:10.1016/S0140-6736(07)61162-3.

Moore, T. M., Reise, S. P., Gur, R. E., Hakonarson, H. y Gur, R. C. (2015). Psychometric properties of the penn computerized neurocognitive battery. Neuropsychology, 29, 235-246. doi:10.1037/neu0000093.

Moreno, L. M. (2018). Informe ESTUDES 2018: Alcohol, tabaco y drogas ilegales en España.

Morton, S. E., O’Hare, K. J. M., Maha, J. L. K., Nicolson, M. P., Machado, L., Topless, R., ... Linscott, R. J. (2017). Testing the validity of taxonic schizotypy using genetic and environmental risk variables. Schizophrenia Bulletin, 43, 633-643. doi:10.1093/schbul/sbw108.

Munafò, M. R., Larsson Lönn, S., Sundquist, J., Sundquist, K. y Kendler, K. (2016). Snus use and risk of schizophrenia and non-affective psychosis. Drug and Alcohol Dependence, 164, 179-182. doi:10.1016/j.drugalcdep.2016.04.035.

Najolia, G. M., Buckner, J. D. y Cohen, A. S. (2012). Cannabis use and schizotypy: The role of social anxiety and 
other negative affective states. Psychiatry Research, 200, 660-668. doi:10.1016/j.psychres.2012.07.042.

Ortuño-Sierra, J., Fonseca-Pedrero, E., Inchausti, F. y Sastre I Riba, S. (2016). Evaluación de dificultades emocionales y comportamentales en población infanto-juvenil: el cuestionario de capacidades y dificultades (SDQ). Papeles del Psicologo, 37, 14-26.

Ortuño-Sierra, J., Fonseca-Pedrero, E., Paino, M., Sastre I Riba, S. y Muñiz, J. (2015). Screening mental health problems during adolescence: psychometric properties of the Spanish version of the strengths and difficulties Questionnaire. Journal of Adolescence, 38, 49-56. doi:10.1016/j.adolescence.2014.11.001.

Pérez Gálvez, M. (2015). Prevención e intervención de la conducta suicida en personas consumidoras de alcohol. En Andoni Aseán (ed.), Manual de prevención, intervención y postvención de la conducta suicida (pp. 611-630). Madrid: Fundación Salud Mental España.

Radua, J., Ramella-Cravaro, V., Ioannidis, J. P. A., Reichenberg, A., Phiphopthatsanee, N., Amir, T., ... Fusar-Poli, P. (2018). What causes psychosis? An umbrella review of risk and protective factors. World Psychiatry, 17, 49-66. doi:10.1002/wps.20490.

Rial, A., Burkhart, G., Isorna, M., Barreiro, C., Varela, J. y Golpe, S. (2018). Cannabis use among adolescents: Risk pattern, implications and possible explanatory variables. Adicciones, 31, 64-77. doi:10.20882/adicciones.1212.

Riala, K., Hakko, H., Isohanni, M., Pouta, A. y Räsänen, P. (2005). Is initiation of smoking associated with the prodromal phase of schizophrenia? Journal of Psychiatry y Neuroscience, 30, 26-32. doi:10.1016/j.psychres.2005.07.014.

Rowe, C., Santos, G.-M., Behar, E. y Coffin, P. O. (2016). Correlates of overdose risk perception among illicit opioid users. Drug and Alcohol Dependence, 159, 234-239. doi:10.1016/j.drugalcdep.2015.12.018.

Saha, S., Scott, J. G., Varghese, D., Degenhardt, L., Slade, T. y McGrath, J. J. (2011). The association between delusional-like experiences, and tobacco, alcohol or cannabis use: a nationwide population-based survey. BMC Psychiatry, 11, 202. doi:10.1186/1471-244X-11-202.

Schubart, C. D., Van Gastel, W. A., Breetvelt, E. J., Beetz, S. L., Ophoff, R. A., Sommer, I. E. C., ... Boks, M. P. M. (2011). Cannabis use at a young age is associated with psychotic experiences. Psychological Medicine, 41, 13011310. doi:10.1017/S003329171000187X.

Sendino, R., Álvares, E., Brime, B., Llorens, N., Ruiz, A. y Sánchez, E. (2016). Informe 2016: alcohol, tabaco y drogas ilegales en España. Observatorio Español de La Droga y Las Toxicomanias. Ministerio de Sanidad y Servicios Sociales, 72. doi:10.1007/s13398-014-0173-7.2.

Shakoor, S., Zavos, H. M. S., Haworth, C. M. A., McGuire, P., Cardno, A. G., Freeman, D. y Ronald, A. (2016). Asso- ciation between stressful life events and psychotic experiences in adolescence: evidence for gene-environment correlations. British Journal of Psychiatry, 208, 532-538. doi:10.1192/bjp.bp.114.159079.

Soto-Brandt, G., Portilla Huidobro, R., Huepe Artigas, D., Rivera-Rei, A., Escobar, M. J., Salas Guzman, N., ... Castillo-Carniglia, A. (2014). Validity evidence of the Alcohol, Smoking and Substance Involvement Screening Test (ASSIST) in Chile. Adicciones, 26, 291-302.

Stewart, D. W., Cohen, A. S. y Copeland, A. L. (2010). Cigarette smoking across the schizotypy spectrum. Psychiatry Research, 179, 113-115. doi:10.1016/J.PSYCHRES.2010.04.038.

Szoke, A., Galliot, A. M., Richard, J. R., Ferchiou, A., Baudin, G., Leboyer, M. y Schürhoff, F. (2014). Association between cannabis use and schizotypal dimensions - A meta-analysis of cross-sectional studies. Psychiatry Research, 219, 58-66. doi:10.1016/j.psychres.2014.05.008.

Van Gastel, W. A., Wigman, J. T. W., Monshouwer, K., Kahn, R. S., Van Os, J., Boks, M. P. M. y Vollebergh, W. A. M. (2012). Cannabis use and subclinical positive psychotic experiences in early adolescence: findings from a Dutch survey. Addiction, 107, 381-387. doi:10.1111/j.13600443.2011.03626.x.

Van Os, J., Linscott, R. J., Myin-Germeys, I., Delespaul, P. y Krabbendam, L. (2009). A systematic review and meta-analysis of the psychosis continuum: evidence for a psychosis proneness-persistence-impairment model of psychotic disorder. Psychological Medicine, 39, 179-195. doi:10.1017/S0033291708003814.

Vázquez, F. y Becoña, E. (2000). Factores de riesgo y escalada cannabinoide. Adicciones, 12 (Supl. 2), 175-184. doi:10.20882/adicciones.680.

Verdoux, H. y Van Os, J. (2002). Psychotic symptoms in non-clinical populations and the continuum of psychosis. Schizophrenia Research, 54, 59-65. doi:10.1016/S09209964(01)00401-7.

Weiser, M., Reichenberg, A., Grotto, I., Yasvitzky, R., Rabinowitz, J., Lubin, G., ... Davidson, M. (2004). Higher rates of cigarette smoking in male adolescents before the onset of schizophrenia: a historical-prospective cohort study. American Journal of Psychiatry, 161, 1219-1223. doi:10.1176/appi.ajp.161.7.1219.

WHO Assist Working Group. (2002). The Alcohol, Smoking and Substance Involvement Screening Test (ASSIST): development, reliability and feasibility. Addiction, 97, 1183-1194. doi:10.1046/j.1360-0443.2002.00185.x.

Zammit, S., Allebeck, P., Dalman, C., Lundberg, I., Hemmingsson, T. y Lewis, G. (2003). Investigating the association between cigarette smoking and schizophrenia in a cohort study. American Journal of Psychiatry, 160, 22162221. doi:10.1176/appi.ajp.160.12.2216. 